\title{
CULTURAS DE PENSAMIENTO PARA EL FORTALECIMIENTO DE HABILIDADES COMUNICATIVAS EN INGLÉS
}

\section{THINKING CULTURES TO STRENGTHEN COMMUNICATION SKILLS IN ENGLISH}

\author{
Sebastian Fernando Marín Hine ${ }^{1}$ \\ Universidad Autónoma de Bucaramanga \\ Elgar Gualdrón Pinto ${ }^{2}$ \\ Universidad de Pamplona \\ Adriana Inés Ávila Zárate ${ }^{3}$ \\ Universidad Autónoma de Bucaramanga
}

\section{RESUMEN}

El presente artículo de investigación tuvo como objetivo el fortalecimiento de las habilidades comunicativas en inglés de niños de transición, a través de la implementación de una secuencia didáctica basada en el enfoque de Pensamiento Visible. El planteamiento metodológico fue de corte cualitativo con

1 *Universidad Autónoma de Bucaramanga (Colombia) smarin63@unab.edu.co ORCID: https://orcid.org/0000-0002-4608-0785

$2 \quad$ Universidad de Pamplona (Colombia) egualdron@unipamplona.edu.co ORCID: https://orcid.org/0000-0002-4081-2092

3 Universidad Autónoma de Bucaramanga (Colombia) aavila2@unab.edu.co

ORCID: https://orcid.org/0000-0002-4122-7960 enfoque de investigación acción en el que se tuvo como muestra un grupo de 15 estudiantes del nivel de transición (entre 5 y 6 años) de un colegio privado de la ciudad de Bucaramanga (Colombia). Los resultados mostraron que la enseñanza del inglés utilizando rutinas de pensamiento contribuyó positivamente a la comprensión del idioma desde su función comunicativa, anteponiéndose al aprendizaje netamente gramatical; se pudo comprobar que la documentación que surgió de las rutinas de pensamiento apoyó el proceso de adquisición del lenguaje, especialmente en lo relacionado con la escritura y la lectura. 


\section{PALABRAS CLAVE:}

adquisición del lenguaje, culturas de pensamiento, lengua extranjera, pensamiento, pensamiento visible, primera infancia

\section{ABSTRACT}

This research article aimed to strengthen the communication skills in English of transitional children, through the implementation of a didactic sequence based on the Visible Thinking approach. The methodological perspective was qualitative with an action research approach in which a group of 15 students of the transition level (between 5 and 6 years) from a private school in the city of Bucaramanga (Colombia) was taken as a sample. The results showed that the teaching of English using Thinking Routines contributed positively to the understanding of the language from its communicative function, taking precedence over purely grammatical learning; it was found that the documentation that emerged from the Thinking Routines supported the language acquisition process, especially in relation to writing and reading.

\section{KEYWORDS:}

language acquisition, cultures of thinking, foreign language, thinking, visible thinking, early childhood

\section{INTRODUCCIÓN}

En el mundo globalizado actual, la comunicación entre fronteras es de gran importancia para el intercambio económico, social, científico, tecnológico, político y cultural de los países (Chávez-Zambrano, SaltosVivas \& Saltos-Dueñas, 2017; Rayón, 2018). El fenómeno de la globalización de acuerdo con Flores (2016) integra a los países en una interdependencia de intercambio social basado en la comunicación, dentro del cual, el lenguaje juega un papel fundamental en el éxito de la incorporación de una nación en el mercado internacional. Desde hace más de 50 años, la lengua elegida para dicho intercambio es el inglés (Richards \& Rodgers, 2014; BBC, 2019; Franco, 2018).

El uso de una lengua mundial para la comunicación entre individuos y naciones de diferentes comunidades lingüísticas no es un fenómeno nuevo ni emergente, por el contrario, se puede rastrear ya desde el milenio IV a.E.C. (antes de la era común) el uso del idioma sumerio como lingua franca (idioma adoptado para un entendimiento común entre personas con diferente lengua materna) el sur de Mesopotamia, gradualmente reemplazado por el idioma arcadio hacia el siglo XIX a.E.C; en la edad del hierro, el arameo antiguo (900-700 a.E.C.) se convirtió en la lengua global de la región conocida como Creciente Fértil, comprendida por los territorios del Levante Mediterráneo (actualmente Israel, Jordania, Líbano, Siria y territorios Palestinos), Mesopotamia y Persia (Baker \& Prys, 1998). Posterior a las conquistas de Alejandro Magno (periodo helenístico), el griego Koiné sirvió como vínculo entre territorios culturalmente diversos que iban desde Roma hasta Egipto, desde la dinastía ptolemaica (323 -30 a.E.C.) hasta el imperio seléucida (312 - 63 a.e.c; Horrocks, 2014). Este recorrido lingüístico recopilado por Baker \& Prys (1998) incluye también al latín (lengua oficial del imperio romano (siglo VIII a.E.C.)) que se mantuvo como lengua científica hasta el siglo XIX; el idioma árabe (s.VI E.C.), la lengua clásica de la India: sánscrito, el idioma chino, el español, el francés, el ruso y actualmente el inglés.

El concepto entonces de una lengua global abarca la necesidad de las civilizaciones por establecer un idioma en común que permita la apertura sociocultural, diplomática y económica entre estas, siendo requisito obligatorio la adquisición de ese idioma como segunda lengua a las comunidades lingüísticas no nativas; "Desde una perspectiva contemporánea e 
histórica, el bilingüismo y multilingüismo es la norma y no la excepción" (Richards \& Rodgers, 2014, p. 1); es también una idea sugerida por Padilla, Aguilar y Manzano (2016).

A pesar de lo anterior, en el territorio colombiano los esfuerzos por desarrollar programas que promuevan la adquisición de una lengua extranjera son relativamente recientes y de poco impacto, tal como lo demuestran los estudios llevados a cabo por la compañía sueca Education First (EF) en 2019. Los resultados obtenidos en la novena edición de la prueba anual EF EPI (English Proficiency Index), que mide el nivel de aptitud en este idioma, ubica a Colombia en el puesto 60 entre 100 países no angloparlantes con un nivel bajo por tercer año consecutivo, subiendo de la categoría "muy bajo" en la que se ubicaba desde el 2011 (EF, 2019). Hay que mencionar que no fue sino hasta el año 2006 cuando el Estado colombiano adoptó el Marco Común Europeo de Referencia para las Lenguas (MCERL) bajo el decreto 3870 y diseñó la Guía 22 con los Estándares Básicos de Competencias en Lenguas Extranjeras (EBCLE): inglés como parte del Programa Nacional de Bilingüismo (2004-2019; MEN, 2006).

Los resultados presentados por EF permiten reflexionar acerca de la metodología con la que se está abordando la enseñanza del inglés; y es que dentro de los planteamientos hechos en la Guía 22 EBCLE, se hace explícita la intención del Ministerio de Educación Nacional de Colombia (MEN) por fomentar una perspectiva funcional del idioma que se fundamente principalmente en la competencia comunicativa, pero entonces ¿por qué no se están logrando los objetivos? ¿por qué los resultados del nivel de aptitud en inglés de los colombianos son tan bajos?

Buscando una aproximación a una posible comprensión de la situación problema, este artículo se propone a mostrar los resultados del proyecto de investigación realizado para fortalecer las habilidades comunicativas de los niños de transición (edades comprendidas entre los 5 y 6 años) de un colegio privado de la ciudad de Bucaramanga (Colombia), utilizando el enfoque de Pensamiento Visible en el que se vinculan los procesos de pensamiento (cognitivos) con el desarrollo del lenguaje. Tal como se expuso anteriormente, el lenguaje es una herramienta comunicativa que permite el intercambio y la interacción entre individuos y culturas, siendo la cultura y el entorno, elementos necesarios para el aprendizaje del ser humano, así como el lenguaje mismo (Vygotsky, 2018). Diferentes perspectivas desde los clásicos de la pedagogía y la psicología como Vygotsky (2018a; 2018b), Piaget (2017; 2019), Bruner (1985; 1996) \&Ausubel (2019), hasta lo expuesto por Dehaene (2019), Damasio (1992) \& Kuhl $(1998 ; 2010)$ en el campo de las neurociencias, coinciden en la relación que existe entre el desarrollo de la inteligencia con la aparición del lenguaje y el rol que tiene la sociedad en este proceso. Las nuevas investigaciones a nivel de neurodesarrollo han demostrado la necesidad de iniciar el proceso de enseñanza de un segundo idioma desde la edad más temprana posible (Costa, 2017; Dehaene, 2019; Gopnik et al., 2000; Lever et al., 2016)

Por lo tanto, si pensamiento y lenguaje están relacionados en el desarrollo del individuo, sería poco provechoso únicamente pensar la enseñanza de una lengua extranjera o segunda lengua desvinculada de un contexto que le permita al estudiante hacer uso de esta herramienta para la comunicación ya que, a la final, ese es el objetivo del lenguaje como tal; la lengua funcional por encima de la instrumental. Lo anterior es de vital importancia para este artículo ya que permite visibilizar dos posibles soluciones a las preguntas planteadas más arriba: 1) La enseñanza del inglés en el país no tiene un objetivo comunicativo sino instrumental (a pesar de lo declarado en los 
documentos del MEN), que únicamente se enfoca en el conocimiento de la gramática para la presentación de pruebas estandarizadas como las pruebas Saber y Saber Pro; 2) No se está aprovechando la etapa sensible que es la primera infancia para fortalecer la adquisición de una segunda lengua a edad temprana; hasta el momento en Colombia no existen lineamientos completos para la enseñanza del inglés en el preescolar a parte de los documentos: Derechos Básicos de Aprendizaje de inglés (DBA) y el Currículo Sugerido para inglés (CSI) propuestos en el año 2016 para transición (MEN, 2016).

Bajo este panorama no es extraño descubrir rendimientos muy bajos en los resultados presentados por EF desde el 2011, el componente gramatical es necesario en el aprendizaje de una nueva lengua, pero, el factor comunicativo no puede ser reemplazado por cumplir una meta que sesga el objetivo final del lenguaje: la efectiva comunicación.

\section{MARCO TEÓRICO}

\section{CULTURA Y SOCIEDAD}

El diccionario de la Real Academia Española (RAE) define la cultura como el "Conjunto de modos de vida y costumbres, conocimientos y grado de desarrollo artístico, científico, industrial, en una época, grupo social, etc." (RAE, 2020). Una segunda definición, que incluye en ella el rol activo en dos vías del individuo en torno a la cultura, proviene del filósofo alemán Johann Gottfried Herder en el que el hombre es además de un ser cultural, un objeto del proceso en sí mismo:

Todo en él es cultivable y requiere ser cultivado, de manera que ni el cuerpo, ni la sensibilidad y las fuerzas del alma son dadas y fijadas sólo por la naturaleza. El hombre no sólo es objeto del proceso cultural, él también es parte del proceso. (Herder en Heinz, 1996, p. 121)
Esta visión activa del individuo dentro del proceso de cultivo cultural tiene muy presente la pertenencia de este a un pueblo que, como explica Heinz (1996), pueblo se entiende como colectividades históricamente unidas por lengua y tradición común por la cual está conformado y creado de determinada manera. La perspectiva de Herder desde la filosofía natural libera al individuo dotándolo de capacidad para culturizarse, es decir aprender; aprender a aprender dentro de la cultura propia de una colectividad (sociedad).

Hablar entonces de cultura implica necesariamente un contrato social activo entre individuos que intercambian los valores más preciados de sus comunidades, entre ellos, las palabras, el lenguaje (Lévi-Strauss, 1981). Con la idea de cultura ya desarrollada y vinculada a la sociedad se hace entonces evidente también, la relación que existe entre el individuo perteneciente a un entorno social, y la influencia que este entorno ejerce sobre él para "culturizarlo"; de esta manera se configura entonces al elemento cultura como un medio educativo, capaz de generar transformaciones internas y externas en el sujeto reguladas por el lenguaje. Lo anterior es lo que Vygotsky (2018a) denominó la teoría sociocultural y de la que identificó dos elementos necesarios para el desarrollo cognitivo del individuo: El pensamiento y el lenguaje.

"el momento más significativo en el curso del desarrollo intelectual, que da a luz las formas más puramente humanas de la inteligencia práctica y abstracta, es cuando el lenguaje y la actividad práctica, dos líneas de desarrollo antes completamente independientes, convergen." (Vygotsky, 2018b, p. 47-48)

Como parte de su trabajo, Vygotsky pone la interacción entre individuos como factor fundamental para el aprendizaje, por lo que 
dentro de su teoría socio cultural propone el concepto de Zona de Desarrollo Próximo (ZDP) que:

“(...) no es otra cosa que la distancia entre el nivel real de desarrollo, determinado por la capacidad de resolver independientemente un problema, y el nivel de desarrollo potencial, determinado a través de la resolución de un problema bajo la guía de un adulto o en colaboración con otro compañero más capaz." (Vygotsky, 2018a, p. 133)

Bajo esta mirada, si el sujeto perteneciente a una sociedad inmersa en una cultura es capaz de culturizar (enseñar) y culturizarse (aprender) mediante la interacción con otros individuos utilizando el recurso del lenguaje (nativo y/o foráneo), se hace apenas lógico pensar en una propuesta que vincule al lenguaje como medio de intercambio que pretenda desarrollar el potencial cognitivo del ser humano, no desde una metodología específica, sino un enfoque de aprender: una cultura de pensamiento.

\section{HACIA UNA CULTURA DEL PENSAMIENTO}

El término Culturas de Pensamiento (del inglés: Cultures of Thinking CoT) es propuesto por Ritchhart (2015) como parte de una de las investigaciones realizadas dentro del grupo Project Zero (PZ) fundado en 1967 por la Escuela de Graduados de Educación de Harvard, con el objetivo de comprender los procesos de aprendizaje en y a través de las artes (Project Zero, 2020). Para Ritchhart (2015) la cultura de pensamiento es la pertenencia a un lugar en el que el pensamiento grupal e individual es valorado, visible y activamente promovido dentro de la experiencia diaria de cada uno de los miembros del grupo; la propuesta del autor está permeada también por las ideas de Perkins (1992) respecto a la creación de escuelas inteligentes, las cuales son definidas como aquellas que se mantienen actualizadas a todo avance producido dentro del campo de la enseñanza y el aprendizaje. Proyectos como Culturas de Pensamiento (Ritchhart, 2015), Escuelas Inteligentes (Perkins, 1992), La guía de la enseñanza para la comprensión (Blythe, 1994), Hacer el aprendizaje visible (Krechevsky et al., 2013) y Pensamiento Visible (Ritchhart et al., 2011) tienen como objetivo hacer de la comprensión el fin último de la enseñanza por medio de la metacognición: Pensar en el pensamiento (Ritchhart, 2002).

Bajo la perspectiva de la Cultura de Pensamiento, la organización de la escuela debe estar enfocada a la construcción de comunidades que "saque lo mejor de cada persona, lleve el aprendizaje al siguiente nivel, permita grandes descubrimientos $\mathrm{e}$ impulse al individuo y al grupo hacia una vida de aprendizaje" (Ritchhart, 2015, p. 6). Para lograr esto, Ritchhart (2015) propone ocho fuerzas (culturales) necesarias para moldear dicha cultura: expectativas, tiempo, modelado, oportunidades, interacciones, ambiente, rutinas y lenguaje, siendo este último la herramienta para la cohesión colectiva y el aprendizaje social. Un contexto de aprendizaje que gire en torno al pensamiento no solo enriquece la experiencia de aprendizaje de los integrantes de dicha comunidad al permitir conocer mejor su funcionamiento cognitivo, sino que, como expone Dehaene (2019), impacta positivamente el éxito escolar.

\section{ADQUISICIÓN Y APRENDIZAJE DE UNA SEGUNDA LENGUA: COMUNICACIÓN}

Como se ha expuesto a lo largo del texto, el lenguaje tiene un lugar muy importante dentro de una comunidad y es también parte de su cultura, no solo para culturizar y culturizarse como mencionaba Herder (Heinz, 1996), sino para intercambiar los valores más preciados de cada uno de estos grupos sociales como añadiría Lévi-Strauss (1981), por lo tanto, para lograr ese intercambio es necesario que la lengua cumpla su función primaria: la comunicación. 
En el abordaje de la enseñanza de una segunda lengua, tal como lo afirma Ordoñez y Rodríguez (2017), bajo estos parámetros, se hace necesario entonces un enfoque que busque el desarrollo de la competencia comunicativa como objetivo principal: el método comunicativo para la enseñanza de la lengua (del inglés: Communicative Language Teaching). Este método busca hacer de la competencia comunicativa el objetivo principal de la enseñanza de idiomas y así desarrollar procedimientos para la adquisición de las cuatro habilidades para la comunicación (escuchar, hablar, escribir y leer) que dan cuenta de la interdependencia del lenguaje y la comunicación (Richard \& Rodgers, 2014). Estas cuatro habilidades de acuerdo con el Enfoque Natural de la Lengua propuesto por Krashen \& Terrell (1998) deberían adquirirse muy rápido siempre y cuando se provea a los estudiantes con contexto comunicativo, “(...) por otro lado, la precisión gramatical aumenta lenta y únicamente después de mucha experiencia utilizando el lenguaje" (Krashen \& Terrell, 1998, p. 16).

El principal error dentro de la enseñanza de una segunda lengua es la falsa creencia de que la comprensión gramatical es un prerrequisito para desarrollar la competencia comunicativa (Krashen \& Terrell, 1998). Tal como se expuso en apartados anteriores el contexto es fundamental para el surgimiento del lenguaje, que visto desde la perspectiva funcional, debe centrarse en el uso de esta dentro de escenarios reales de comunicación; estos contextos entendidos para el proyecto de investigación como comunidades académicas (comunidad escolar/ clases de inglés) deben organizarse en torno a la experiencia del uso real de la lengua como herramienta de interacción (de intercambio), tal como lo hace el niño cuando aprende su lengua materna (Kuhl, 1998; Dehaene, 2019), de forma natural (Krashen \& Terrell, 1998; Costa, 2017).

\section{PRIMERA INFANCIA}

Estudios recientes en el campo de la neurociencia demuestran la importancia que tiene la exposición del niño a una segunda lengua desde los primeros momentos de vida (Werker \& Hensch, 2014; Yang, 2013; Dehaene, 2018; Kuhl, 2010). Lo anterior no significa que, si no se hace, el individuo no pueda más tarde adquirir un nuevo idioma, pero si se resalta la diferencia entre velocidad de adquisición, desarrollo de una buena pronunciación y capacidad de uso (Dehaene, 2019).

Vygotsky (2018b) ya había abordado el aprendizaje de una segunda lengua afirmando que la adquisición de un idioma nuevo se nutre principalmente de los conocimientos y conceptos adquiridos en la lengua nativa por un proceso de transferencia. La profesora Kuhl (2010) demostró que, aunque este proceso es visible en regiones monolingües, no engloba el fenómeno del bilingüismo en sí, ya que estudios realizados en este campo comprueban que todo individuo durante los primeros días de nacido puede identificar y aprender los matices más sutiles entre diversas lenguas, pudiendo reorganizar las conexiones de sus cerebros (Costa, 2017), para adquirir dos o más idiomas siempre y cuando el contexto lo provea de entornos de comunicación y uso activo de las lenguas.

Este proceso de adquisición de una segunda lengua a muy temprana edad coincide con el enfoque Natural para la enseñanza del lenguaje de Krashen \& Terrell, principalmente soportado por la plasticidad cerebral que, definida por Dehaene (2019), es la capacidad que tiene el cerebro para formar nuevas conexiones y eliminar aquellas que no se estimulan más. La plasticidad cerebral no es una capacidad que se pierda del todo con los años, pero si está en su punto álgido durante el periodo sensible denominada como primera infancia que abarca desde el nacimiento hasta 
los ocho años (Piaget, 2017). Las sinapsis se modifican constantemente a lo largo de la vida; estos cambios son el reflejo de nuestro aprendizaje (Takeuchi et al., 2014).

Esta nueva revisión sobre la forma en que el niño aprende permite hacer visible la importancia que tiene el periodo preescolar para el desarrollo del ser humano, ya no solo a nivel de lenguaje, sino a nivel cognitivo en general en el que se urge a la comunidad (social, educativa) a replantear la manera de enseñar y de organizar sus currículos.

\section{METODOLOGÍA}

La investigación realizada se ubicó dentro del paradigma cualitativo, específicamente bajo los principios de la investigación-acción definida por Kemmis (1980) como:

"(...) una forma de búsqueda autorreflexiva, llevada a cabo por participantes en situaciones sociales, para perfeccionar la lógica y la equidad de las propias prácticas sociales o educativas, la comprensión de estas prácticas y las situaciones en las que se efectúan estas prácticas." (Kemmis, 1980, p. 30)

La implementación del proyecto tuvo en cuenta las fases de investigación propuestas por Kemmis \& McTaggart (1992) en las que identifican en primer lugar la fase de planeación, seguida por el actuar, el observar y finalmente reflexionar. Buscando abordar el objetivo propuesto de fortalecer las habilidades comunicativas en inglés de niños de transición, se diseñó una secuencia didáctica basada en el enfoque de Pensamiento Visible (enfoque compatible con las culturas de pensamiento) con el fin de vincular la adquisición de una segunda lengua con el desarrollo cognitivo.

La recolección de los datos para el posterior análisis se hizo a través de la observación participante, así como el registro de notas de campo, producciones escritas de los estudiantes y registro fotográfico y de video. Otro recurso importante tenido en cuenta para la recolección de la información fueron las rutinas de pensamiento, herramientas propias del enfoque de Pensamiento Visible.

La intervención de corte cualitativo tuvo lugar en un colegio privado para la primera infancia en la ciudad de Bucaramanga (Colombia, en la que participaron 15 estudiantes del nivel de transición con edades entre los 5 y 6 años. La institución implementa el currículo internacional propuesto desde el modelo VESS (Vida Equilibrada con Sentido y Sabiduría) en el que además del enfoque de Pensamiento Visible confluyen diferentes teorías en torno al desarrollo y la utilización del pensamiento como herramienta pedagógica materializadas en diferentes módulos a modo de proyectos.

\section{ACERCA DEL PROBLEMA DE INVESTIGACIÓN}

Cuando la enseñanza del inglés debe responder a factores instrumentales de la lengua (resolver tests, generalmente escritos), se desvirtúa el carácter funcional de la misma (comunicativo), privilegiando entonces algunas de las habilidades comunicativas para dicho fin: escritura y lectura. Este sesgo en el aprendizaje de una nueva lengua permite que los estudiantes tengan un conocimiento consciente de las reglas gramaticales pudiendo mediante la lógica rellenar verbos faltantes, intuir pronombres personales e incluso seleccionar entre varias opciones la respuesta correcta a la pregunta. Desafortunadamente, este enfoque basado en la gramática no permite que los aprendices lleven al ámbito comunicativo dichas reglas interiorizadas quedando atrapados únicamente en respuestas estereotipadas a preguntas estandarizadas como, por ejemplo: What's your name?; What day is today?; How is the weather?; How old are you? entre otras. 
Este fenómeno ya expuesto al inicio del artículo, a nivel de país, también afectó a la institución privada en donde se desarrolló la investigación; comopartedelapromesaeducativa del colegio, los estudiantes de transición deben poder resolver satisfactoriamente las pruebas de admisión en colegios generalmente bilingües o que tienen un mayor énfasis en esa lengua debido a que la institución no ofrece educación primaria, solamente preescolar. Con el fin de cumplir dicho requerimiento las clases de inglés se orientan al almacenamiento de vocabulario por temas específicos: animales, profesiones, partes de la casa, partes del cuerpo, colores, formas, entre otros; también se hace énfasis en el correcto posicionamiento de las diferentes partes de la oración, pronombres personales, tiempos verbales, asociación de palabra-imagen y conteo mecánico hasta el 100 ya que en eso es que se enfocan dichas pruebas de admisión en su parte escrita.

La parte verbal, si la hay, es la respuesta automatizada a preguntas estandarizadas, $y$, se denominaron estandarizadas debido a que funcionan como una fórmula exacta, por ejemplo, si en una conversación el entrevistador quisiera conocer sobre el estado general del estudiante tendría que preguntar: How are you? para recibir como respuesta -I am (happy/ fine/etc), pero si el entrevistador aun teniendo como objetivo conocer el estado del estudiante le preguntara: How are you doing?; What's up?; How's it going?; How's everything? muy probablemente la respuesta no se genere aunque todas las producciones anteriores sean variables de la pregunta inicial. Este problema se debe principalmente a la presión que tienen las instituciones por mostrar resultados (producciones) sin que necesariamente exista una comprensión y/o adquisición de la lengua; el estudiante trabaja únicamente desde la memoria, la lógica y la asociación (instrumental) y no funcional (comunicativa).
Finalmente, es necesario decir que a pesar de que la institución a nivel curricular utiliza el modelo VESS, el cual pone al pensamiento como herramienta pedagógica, el área de inglés no está alineada a dicha filosofía. Lo anterior se debe principalmente a que la enseñanza de una lengua extranjera se percibe como una asignatura aparte, individual, que se centra en la producción del idioma a nivel gramatical pero que no lo relaciona con otros procesos cognitivos, es decir, se rompe el vínculo entre el lenguaje y el pensamiento. Como resultado de esta problemática surgen dos preguntas:

\ ¿Cómo fortalecer las habilidades comunicativas en inglés de los niños de transición de un colegio privado en la ciudad de Bucaramanga (Colombia) con actividades que ayuden a su vez a desarrollar habilidades de pensamiento?

$\otimes$ ¿Cómo generar un espacio dentro de la clase de inglés para niños de transición en donde la cultura de pensamiento permita el fortalecimiento de las habilidades comunicativas en inglés?

\section{DISEÑO DE LA SECUENCIA DIDÁCTICA A PARTIR DE LAS FUERZAS CULTURALES}

Una secuencia didáctica es "(...) la organización de las actividades de aprendizaje que se realizarán con los alumnos y para los alumnos con la finalidad de crear situaciones que les permitan desarrollar un aprendizaje significativo" (Díaz-Barriga, 2013 p. 2). Como se expuso desde el marco teórico, a nivel de la enseñanza de la lengua, las actividades propuestas deben responder a la generación de contextos reales de comunicación donde el lenguaje sea visto como una herramienta de intercambio que, como añadidura a la definición de secuencia didáctica, deben ser significativas:

“(...) hay aprendizaje significativo si la tarea de aprendizaje puede relacionarse, de 
modo no arbitrario y sustancial (no al pie de la letra), con lo que el alumno ya sabe y si éste adopta la actitud de aprendizaje correspondiente para hacerlo así." (Ausubel et al., 2019, p. 37)

Con respecto al aprendizaje de una segunda lengua, bajo la mirada del aprendizaje significativo, Ausubel et al. (2019) hablan de establecer un equivalente representacional entre las palabras del segundo idioma y las palabras ya establecidas en el lenguaje natal del alumno, una afirmación basada en el pensamiento de Vygotsky (2018b). Teniendo esto en cuenta, la secuencia didáctica se diseñó en torno al contenido My body (mi cuerpo), ya que era la temática establecida desde el currículo para el periodo en que se realizó la intervención, siendo un eje temático meramente circunstancial. La intervención se hizo en 15 sesiones que tenían relación entre ellas, utilizando las rutinas (una de las fuerzas culturales) en forma de rutinas de pensamiento para exteriorizar el proceso de aprendizaje. Cada actividad se configuró bajo los preceptos de las fuerzas culturales, el enfoque Natural para la enseñanza de la lengua y Pensamiento Visible.

Las categorías de análisis establecidas respondieron al objetivo general del proyecto de investigación que buscó fortalecer las habilidades comunicativas en inglés de los niños de transición, por lo que dichas categorías salen del Marco Común Europeo de Referencia para las Lenguas (MCERL), tomando como base el nivel de referencia A1 (acceso). En la tabla 1 se presentan las tres categorías de análisis: Comprensión $(C)$, hablar $(H)$ y escribir $(E)$, cada uno con sus subcategorías: Escucha (CE), lectura (CL), monólogos (HM), conversación $(\mathrm{HC})$ y escritura (EE).

Tabla 1.

\begin{tabular}{|c|c|c|c|c|}
\hline \multicolumn{5}{|c|}{ Categorías de análisis } \\
\hline Com & 1sión (C) & $\mathrm{Habl}$ & & Escribir (E) \\
\hline $\begin{array}{l}\text { Escucha } \\
\text { (CE) }\end{array}$ & Lectura (CL) & Monólogos (HM) & $\begin{array}{c}\text { Conversación } \\
(\mathrm{HC})\end{array}$ & Escritura (EE) \\
\hline
\end{tabular}

Fuente: Elaboración propia

\section{ANÁLISIS DE LA INTERVENCIÓN}

\section{DIAGNÓSTICO}

El diagnóstico de las habilidades comunicativas se hizo a partir de la implementación en tres sesiones de dos rutinas de pensamiento: Zoom in, I see I think y nuevamente Zoom in. Los resultados obtenidos permitieron evidenciar, dentro de la categoría (C), una comprensión parcial del contenido e instrucciones básicas siempre y cuando el profesor se apoyara en gestos, ayudas visuales y/o traducción; este nivel de comprensión afectó directamente la participación y por ende el intercambio de ideas $(H)$. Durante las dos primeras sesiones se observó que la producción oral (H) estaba limitada a respuestas no verbales en aquellos estudiantes que lograban comprender la instrucción (mayoría en las dos primeras sesiones) y un rechazo a participar en aquellos que definitivamente no comprendían inicialmente la actividad.

Es notable resaltar que, durante la tercera sesión, el número de estudiantes que no comprendieron ni participaron se redujo a cero y que la comprensión general del salón aumentó significativamente en parte a que la rutina utilizada (Zoom In) ya se había trabajado 
en la sesión uno. Lo anterior se atribuye al manejo previo de la estructura de la rutina de pensamiento, en la que los estudiantes ya no tuvieron que preocuparse por tres elementos: funcionamiento de la rutina, contenido y lenguaje sino únicamente por los dos últimos. Hay que mencionar otro factor que permitió la mejoría en la sesión tres y es la permisión (no explícita) de la lengua materna como andamio lingüístico (Wood, Bruner \& Ross, 1976).

\section{ANÁLISIS DE LA INTERVENCIÓN}

\section{HABILIDADES COMUNICATIVAS}

Los datos obtenidos en cada sesión se recogieron dentro de rúbricas, producciones escritas y transcripciones de conversaciones entre estudiante-profesor y estudianteestudiante.
Tal como se mencionó anteriormente, dentro de las diferentes actividades se permitió el uso implícito de la lengua materna como andamio lingüístico y conceptual para la lengua extranjera; esta decisión se inspiró en el trabajo realizado por Salmon (2008) en el que se soporta la importancia de la lengua nativa en la construcción de un nuevo idioma (bilingüismo aditivo; Lambert, 1974) a través del uso de rutinas de pensamiento. Esta nueva permisión condujo a una mayor participación de los estudiantes en cada una de las sesiones, tal como se aprecia en la tabla 2 en la que se relaciona el número de estudiantes que participaron de las diferentes sesiones, con los descriptores establecidos en la rúbrica de evaluación.

Tabla 2.

Relación participación estudiantes por sesión de acuerdo a descriptores de rúbrica.
Sesiones/Participación estudiantes

$\begin{array}{rrrrrrrrrrrrrr}1 . & 2 . & 3 . & 4 . & 5 . & 6 . & 7 . & 8 . & 9 . & 10 . & 11 . & 12 . & 13 . & 14 .\end{array}$

\begin{tabular}{llllllllllllllll}
\hline $\mathbf{1}^{*}$ & 4 & 5 & 3 & 4 & 4 & 7 & 6 & 6 & 7 & 7 & 8 & 8 & 7 & 8 & 8 \\
\hline $2^{*}$ & 6 & 5 & 7 & 6 & 6 & 5 & 6 & 6 & 7 & 8 & 4 & 6 & 6 & 7 & 7 \\
\hline $3^{*}$ & 3 & 3 & 4 & 4 & 4 & 3 & 3 & 1 & 1 & 0 & 3 & 1 & 2 & 0 & 0 \\
\hline $4^{*}$ & 2 & 2 & 1 & 1 & 1 & 0 & 0 & 2 & 0 & 0 & 0 & 0 & 0 & 0 & 0
\end{tabular}

\section{Descriptores}

$1^{*}$. Participa de manera activa dentro de la rutina. Quiere participar de primero. Demuestra interés por lo que se está haciendo. Motiva y da apoyo a los compañeros que están participando. Ayuda con la organización de la actividad. Se mantiene conectado hasta el final.

2*. Participa voluntariamente en la rutina. Demuestra interés hacia lo que se está haciendo. Permanece atento durante la rutina.

$3^{*}$. Participa únicamente cuando se le pide que participe. Su nivel de interés varía. Se desconecta fácilmente de la actividad.

$4^{*}$. No participa de la rutina. Le es difícil mantenerse enfocado en la actividad. Se distrae con facilidad. 
El aumento significativo en la participación también redujo el número de estudiantes que no se conectaban con las diferentes actividades; este fenómeno trajo consigo un incremento en la producción oral para la interacción estudianteprofesor dentro de las rutinas. Al poder utilizar los conceptos que ya poseía en español, le era mucho más sencillo al niño transferirlo al inglés y reemplazarlo dentro de una conversación simple. La siguiente transcripción es un ejemplo tomado de la sesión 4 entre una estudiante $(E)$ y el profesor $(P)$ mientras coloreaban al elefante Elmer.

-P: What are you doing?

-E: Elmer the elephant!

-P: And how is he? What did you do?

-E: He is big... con puntos red en la espalda y las piernas. La ear is yellow y la cara pink. on his nose?

-P: Great! And what about all those color arcoíris.

-E: Es que quería hacerlo como un

-P: Do you mean a rainbow, right?

-E: Si, un rainbow de muchos colores.

-P: It's amazing! Great job!

-E: Thank you.
Las interacciones llevadas a cabo durante la intervención demostraron una mejora en los niveles de comprensión dentro de una conversación, además de la producción de oraciones híbridas (español-inglés) que buscaban hacer partícipe al estudiante de la conversación como agente comunicativo. Con el diseño de las actividades, bajo los lineamientos del enfoque de Pensamiento Visible y los materiales sugeridos desde el enfoque Natural para la adquisición de la lengua, se pudo observar que el uso de realia (objetos utilizados para el aprendizaje, sin ser esta su naturaleza primaria) fortaleció los procesos de apropiación de contenidos y vocabulario mucho más rápido que la simple repetición o presentación abstracta de los mismos; esta decisión benefició también ampliamente la documentación dentro de las rutinas de pensamiento haciendo aún más explícito lo que los estudiantes estaban aprendiendo al tener que registrar sus ideas de manera escrita y/o gráfica.

En la tabla 3 se presentan los datos obtenidos dentro de cada una de las sesiones realizadas haciendo énfasis en el tipo de producción oral presentado por los estudiantes. Se pudo observar un aumento significativo a nivel de producción/participación utilizando el inglés como se indica en los descriptores 2 y 3 , logrando pasar de respuestas gestuales y sin estructura a participaciones sencillas pero organizadas.

Tabla 3. 


\section{Descriptores}

$1^{*}$. Se comunica en inglés, y en caso de no tener el vocabulario necesario, se apoya en movimientos y/o gestos que complementen su idea, evitando al máximo hacer uso de la lengua materna.

$2^{*}$. Se comunica verbalmente, utilizando en gran medida su lengua materna, únicamente traduciendo al inglés el vocabulario clave e insertándolo dentro de una oración Ej: El carro es red.

$3^{*}$. Se comunica en gran medida haciendo uso de gestos y movimientos corporales, algunas veces acompañándolos de palabras sueltas en inglés. No hay estructuras. Ej: Yellow

4*. Se comunica únicamente en su lengua materna.

\section{DOCUMENTAR: ESCRIBIR Y LEER}

El uso de rutinas de pensamiento por sí solas no promueven los procesos de lectura y escritura de los estudiantes, en cambio sí permiten un acercamiento a través de la escritura creativa al reconocimiento gráfico de la fonética del inglés. Habiendo afirmado lo anterior, es necesario decir que, para que este reconocimiento se dé, debe existir un trabajo paralelo en lo referente a la conciencia fonológica ya que por la edad y el proceso de lectura y escritura que llevan los niños en su lengua materna pueden surgir confusiones que retrasen esta adquisición. En la institución donde se llevó a cabo la intervención, los niños inician el estudio de la conciencia fonológica en inglés desde el nivel de jardín.

En la tabla 4 se muestran los resultados a modo de comparación de las producciones escritas contrastadas con la palabra correcta en inglés; es necesario decir que los estudiantes

Tabla 4

\begin{tabular}{cc}
\hline \multicolumn{2}{c}{ Producciones escritura creativa } \\
\hline Escritura creativa & Palabra correcta \\
\hline iex & Eyes \\
\hline teef & Teeth \\
\hline hort & Heart \\
\hline jed & Head \\
\hline
\end{tabular}

Nota: elaboración propia 
De igual forma hubo estudiantes con producciones literales en las que la pronunciación de la palabra en inglés era adaptada a los fonemas del español tal como se presenta en la tabla 5 .

Tabla 5

Producciones escritura literal

\begin{tabular}{|c|c|}
\hline Producción estudiante & Palabra correcta \\
\hline elbou & elbow \\
\hline touz & Toes \\
\hline jan & Hand \\
\hline Pinas & Penis \\
\hline
\end{tabular}

Con respecto a la habilidad de lectura, tal como se mencionó anteriormente, las rutinas de pensamiento por sí solas no contribuyen a su adquisición, pero si funciona como una herramienta monitor; es decir, un espacio físico presente dentro del salón de clase en el que el estudiante puede consultar en cualquier momento el vocabulario visto durante los diferentes momentos de la experiencia de aprendizaje entre sesiones y complementar, corregir y/o recuperar saberes. Hacer el pensamiento visible a través de las rutinas de pensamiento crea espacios para la socialización de los individuos pertenecientes a la cultura de pensamiento, pero también a aquellos observadores externos interesados en comprender el proceso de aprendizaje.

\section{CONCLUSIONES}

El lenguaje como herramienta social y cognitiva debe enseñarse bajo la visión de elemento funcional en entornos que permitan el uso de esta dentro de situaciones comunicativas y no meramente instrumentales. Buscando el fortalecimiento de las habilidades comunicativas en inglés, los resultados de esta intervención permitieron comprobar los aportes para la adquisición del inglés que ofrecen los enfoques basados en la metacognición, en este caso puntual el enfoque de Pensamiento Visible integrado al Enfoque Natural de la Lengua.

La integración de estos dos puntos de vista dentro de la secuencia didáctica permitió mejorar la calidad del aprendizaje, la participación del estudiante y el uso de la nueva lengua de forma transversal con contenidos cotidianos y fáciles de conectar; haciendo del proceso de adquisición lingüístico una experiencia significativa, fácilmente expandible a cualquier temática. Esta dinámica de enseñanza y aprendizaje dentro de las clases de inglés permitió repensar la clase de lenguas como un curso aislado, centrado únicamente en la gramática y el vocabulario; si el lenguaje es efectivamente un puente entre el individuo y el entorno, el aprendizaje de una segunda lengua debe ser visto de la misma manera y que además aporta un componente cultural y social adicional al aprendizaje del niño, permitiéndole ampliar su rango de influencia al poderse comunicar en otro idioma.

\section{EL ESTUDIO LLEVADO A CABO PERMITE SUGERIR:}

1. La propuesta pedagógica basada en hacer visible el pensamiento promovió el fortalecimiento de todas las habilidades comunicativas en inglés ya que, a medida que 
el estudiante interactuaba con el profesor y sus compañeros, trabajaba la habilidad de escucha (listening) y habla (speaking); cuando tuvo que documentar su pensamiento (en el caso de ser escrito), fortalecía la escritura (writing), ya sea transcripción y/o escritura creativa. Finalmente, al tener que revisar la documentación para retomar ideas previas y plantear nuevas, hizo uso de la habilidad de lectura (reading).

2. La enseñanza de una segunda lengua debe partir de una visión funcional del idioma y no una instrumental; cuando el estudiante logra conectar el nuevo vocabulario y estructuras gramaticales a sus pre saberes, el proceso de adquisición es mucho más natural y duradero que un enfoque por repetición.

3. Se deberían diseñar entornos de aprendizaje centrados en la participación e intercambio de ideas (comunicación) que permitan dejar registro físico de dichas interacciones, ya que como se mencionó anteriormente, la documentación funciona como andamio emocional y cognitivo para futuras sesiones al poder ser visitados nuevamente. El enfoque de Pensamiento Visible con las rutinas de pensamiento ayuda a que los estudiantes tengan a su disposición todo el contenido enseñado y pueda relacionarlo fácilmente con nuevos temas y/o recurrir a estas en caso de tener dudas o rectificar.

4. Con respecto de la lectura y escritura en inglés, se evidenció que el avance de estas dos habilidades está directamente relacionado con los mismos procesos llevados por el niño en su lengua materna, sin que esta afirmación sugiera que, porque un estudiante sea capaz de leer en español, automáticamente va a leer en inglés. Lo que este estudio permitió evidenciar es que, al llevar el estudiante un buen proceso de lectura y escritura en español, le facilitará el acceso al material escrito en inglés, dependiendo del nivel de reconocimiento fonético de la lengua extranjera que posea.

5. Dentro del ámbito educativo, donde el aprendizaje es el resultado de la interacción social de un individuo con personas más capaces, el lenguaje funge como el puente cognitivo entre el mundo externo $y$ el desarrollo interno (cognitivo) de ese individuo.

6. La comunidad escolar (escuelas) al ser entornos que promueven el aprendizaje a través de clases estructuradas, deben velar por la formación de un modo de ser que refleje los objetivos en torno al desarrollo cognitivo de sus estudiantes: una cultura de pensamiento en la que los elementos a intercambiar sean producto de un ambiente diseñado para la generación del pensamiento y que la adquisición de una nueva lengua se integre a dicha dinámica.

7. Mediante el diseño de la secuencia didáctica se evidenció que, al desarrollar actividades que permitan al estudiante conectarse de múltiples formas con el mensaje (contenido) dado por el profesor (input), además de poder hacer uso de su lengua materna para expresar sus ideas, no solo aumenta los niveles de participación dentro de las actividades, sino que además enriquece la adquisición del conocimiento, la comprensión del mismo y la exteriorización de ese pensamiento utilizando diferentes maneras para documentarlo. 


\section{REFERENCIAS}

Ausubel, D., Novak, J. \& Hanesian, H. (2019). Psicología educativa: Un punto de vista de cognoscitivo ( $2^{\mathrm{a}}$ ed.). Editorial Trillas, S.A.

Baker, C. \& Prys, S. (1998) Encyclopedia of Bilingualism and bilingual education. Multilingual Matters.

BBC News Mundo (2019). Cómo el inglés se convirtió en la lengua "universal" de la ciencia. https://www.bbc.com/mundo/ noticias-49610224

Bruner, J. (1985). Child's talk (1 ${ }^{\text {a }}$ ed.). W.W. Norton \& Company Ltd.

Bruner, J. (1996). The culture of education (1 ${ }^{\mathrm{a}}$ ed.). Harvard University Press.

Chávez-Zambrano, M., Saltos-Vivas, M. \& SaltosDueñas, C. (2017). La importancia del aprendizaje y conocimiento del idioma inglés en la educación superior. Dom. Cien., 3(3), 759-771. https://doi. org/10.23857/pocaip

Costa, A. (2017). El cerebro bilingüe: La neurociencia del lenguaje ( $1^{\mathrm{a}} \mathrm{ed}$.). Editorial Debate.

Damasio, A., \& Damasio, H. (1992). Brain and language. Scientific American, 267(3), 63-71. https://doi.org/10.1038/ scientificamerican0992-88

Dehaene, S. (2019). ¿Cómo aprendemos? (1er ed.). Siglo Veintiuno Editores.

Franco, E. (2018). Daniel Dejica, Gyde Hansen, Peter Sandrini \& Lulia Para (eds.). Languaje in the Digital Era. Challenges and perspectives. De Gruyter Open, Berlin, 2016: 270 pp. Nueva Revista de Filosofía Hispánica, LXVI (1); 203-210.
Gopnik, A., Meltzoff, A. \& Kuhl, P. (2000). The scientist in the crib: What early learning tells us about the mind ( $2^{\mathrm{a}}$ ed.). William Morrow Paperbacks.

Heinz, M. (1996). Kulturtheorien der Aufklãrung. En Nationen und kulturen: zum 250. Geburtstag Johann Gottfried Herders (1ª ed., pp. 139-152). Königshausen \& Neumann.

Horrocks, G. (2014) Greek: A history of the language and its speakers ( $2^{\circ}$ ed.). Wiley-Blackwell

Kemmis, S. (1980). Action Research in Retrospect and Prospect. Autralian Association for Research in Education.

Kemmis, S., \& McTaggart, R. (1992). Cómo planificar la investigación-acción. Editorial Laertes.

Kuhl, P. (2010). Brain mechanisms in early language acquisition. Neuron, 67(5), 713-727. https://doi.org/10.1016/j. neuron.2010.08.038

Lambert, W. (1974). Culture and language as factors in learning and education. En Cultural factors in learning. F. Aboud (ed.).

Lever, J., Montes de Oca, M., Polo, A., \& Victorio, A. (2016). Factores explicativos de rendimiento académico en hijos de inmigrantes mexicanos en Nueva York. Psicología Educativa. Revista de los Psicólogos de la Educación, 22(2), 125133.

Lévi-Strauss, C. (1981). Las estructuras elementales del parentesco. Editorial Páidos. 
Ordoñez, M. \& Rodríguez, J. (2017). Análisis de las estrategias e instrumentos de evaluación en el aprendizaje del inglés como lengua extranjera. Ideales, 6,81 94.

Padilla, D., Aguilar, J. M., \& Manzano, A. (2016). Translanguaging como estrategia de aprendizaje de L2. International Journal of Developmental and Educational Psychology, 1 (1), 307-314.

Piaget, J. (2017). 6 estudios de psicología (19a ed.). Editorial SKALA.

Piaget, J. (2019). Piscología y pedagogía: cómo llevar la teoría del aprendizaje a la práctica docente. Siglo XXI editores.

Rayón, M. C. (2018). La globalización: su impacto en el Estado-Nación y en el Derecho. Revista jurídica derecho, 7(8), 19-37.

Richards, J., \& Rodgers, T. (2014). Approaches and methods in language teaching $\left(3^{\mathrm{a}}\right.$ ed.). Cambridge University Press.

Ritchhart, R. (2015). Creating cultures of thinking: The 8 forces we must master to truly transform our schools ( $1^{\text {a }}$ ed.). Jossey-Bass.

Ritchhart, R., Church, M., \& Morrison, K. (2011). Making Thinking Visible ( $1^{\mathrm{a}}$ ed.). JosseyBass.

Salmon, A. (2008). Young English language learners making thinking and language visible. Colombian Applied Linguistics Journal, (10), 126-141. https://doi. org/10.14483/22487085.101

Siegel, J. (1985) Koines and koineization. Cambridge University Press, 14(3), 357-378. https://doi.org/10.1017/ $\underline{\mathrm{S} 0047404500011313}$
Takeuchi, T., Duszkiewics, A. \& Morris, R. (2014). The synaptic plasticity and memory hypothesis: Encoding, storage, and persistence. Philosophical Transactions of the Royal Society B, 396(1633). https://doi.org/10.1098/rstb.2013.0288

Vygotsky, L. (2018a). El desarrollo de los procesos psicológicos superiores $\left(4^{\mathrm{a}} \mathrm{ed}\right.$.). Crítica.

Vygotsky, L. (2018b). Pensamiento y lenguaje ( $3^{\mathrm{a}}$ ed.). Ediciones culturales Paidós, S.A.

Werker, J. \& Hensch, T. (2014). Critical periods in speech perception: New directions. Annual Review of Psychology, 66(1), 173-196. https://doi.org/10.1146/ annurev-psych-010814-015104

Wood, D., Bruner, J. \& Ross, G. (1976). The role of tutoring in problem solving. The Journal of child psychology, 2(17), 89-100. https:// doi.org/10.1111/j.1469-7610.1976. tb00381.x

Yang, C. (2013). Ontogeny and phylogeny of language. Proceedings of the National Academy of Sciences of the United States of America PNAS, 110(16), 6324-6327. https://doi.org/10.1073/ pnas. 1216803110 